\title{
Atmospheric Effect on Cosmic Ray Muons at High Cut-Off Rigidity Station
}

\author{
Abdullrahman Maghrabi and Mohammed Almutayri \\ National Centre for Applied Physics, King Abdulaziz City for Science and Technology, Riyadh 11442, Saudi Arabia \\ Correspondence should be addressed to Abdullrahman Maghrabi; amaghrabi@kacst.edu.sa
}

Received 1 December 2015; Revised 14 March 2016; Accepted 27 March 2016

Academic Editor: Valery Nakariakov

Copyright (C) 2016 A. Maghrabi and M. Almutayri. This is an open access article distributed under the Creative Commons Attribution License, which permits unrestricted use, distribution, and reproduction in any medium, provided the original work is properly cited.

\begin{abstract}
Cosmic ray data and radiosonde measurements from Riyadh, Saudi Arabia (Rc=14.4 GV), for the period 2002-2012, were used to study the effect of atmospheric pressure, level of pion production, and temperature at that level, on cosmic ray muons. We found that, even if corrections were made to the detected muons using these three parameters, seasonal variations of the cosmic rays still exist. This suggests that other terrestrial and/or extraterrestrial causes may be considered. The levels of pion production and atmospheric pressure are inversely correlated with the muon rate. On the other hand, the temperature at the pion production level is correlated with muons in spring and winter and inversely correlated in fall and summer. There is no clear explanation for this behavior.
\end{abstract}

\section{Introduction}

Recently it has been found that the influence of solar and heliospheric processes on the primary cosmic ray incident at the top of the atmosphere leads to changes in some atmospheric properties, which in turn affect global weather and climate [1-6].

To be able to study cosmic rays and their variations in a proper way, using ground level detectors, atmospheric effects on secondary cosmic rays must be removed [7-10]. These secondary particles result from the interaction of the primaries with atmospheric nuclei, in which muons are considered the majority of these particles detected at sea level.

The rate of the detected muons depends on some atmospheric factors; corrections for local variations of these factors must be made to determine the properties of the primary cosmic rays. According to theoretical and experimental investigations, atmospheric pressure is the most important factor affecting both cosmic ray muons and neutrons. It is a measure of the total atmospheric absorption above the detector (e.g., $[8,11-13])$.

Atmospheric temperature is the second factor affecting, particularly, cosmic ray muons. The temperature correction is rather complicated and several methods have been developed to account for it. Some workers have used the temperature at screen levels [14-18]; others used the weighted temperature, which is a measure of temperature over the whole atmospheric profile $[12,13,19]$, while still others used the temperature at the pion production level [20]. It has been found (e.g., $[12,13,15])$ that the method of weighted temperature (i.e., the integration method) is the most reasonable procedure to correct for the temperature effect on cosmic ray muons. However, due to the scarcity of the atmospheric temperature measurements at certain atmospheric levels or for the whole profile, this method has been implemented less. The third common factor used by some investigators was the level of pion production [9]. In correcting for cosmic ray muons, workers have used these factors interchangeably [15].

This paper is a continuation of our work to study the effect of atmospheric variables on the detected cosmic ray muons at a midlatitude city.

In this paper, we use 11 years of muon measurements collected by the KACST detector to study the influence of atmospheric pressure, level of pion production, and the temperature at that level, on the detected muons, and determine the appropriate correction coefficients. 


\section{Instrumentation and Methods}

Cosmic ray muon data were obtained from the King Abdulaziz City for Science and Technology (KACST) detector for the period 2002-2012. The detector is a $1 \mathrm{~m}^{2}$ plastic scintillator and viewed photomultiplier tube (PMT), both contained in a light-tight box. The signals from the PMT are preamplified, amplified, and digitized by an Analogue to Digital Converter (ADC). These electronic circuits and temperature and pressure sensors are designed as part of a collaborative project with the University of Adelaide, Australia. Detailed descriptions for this detector and calibration procedures are given elsewhere (Maghrabi et al., 2011) [17, 18]. The detector was installed in the fourth building of KACST's main building and has been in operation since July 2002. However, during this period, the detector went through periods of downtime for calibration procedures, relocations, and power failure. These cause some periods of missing data. For the purpose of this study, we believe that these missing data will not affect the obtained results.

Radiosonde data from Riyadh airport for the corresponding period of time were obtained from the Saudi Presidency of Meteorological Environment (PME). Usually radiosonde measurements are conducted twice a day; however, there were some days when three or more measurements were available. Theoretical and experimental results carried out by several investigators (e.g., [8]) have shown that the pion production level is usually between 70 and $200 \mathrm{mb}$. Following the procedure adapted by several investigators (e.g., [9]) we have assumed $100 \mathrm{mb}$ as the pion production level. From available data, atmospheric temperatures and the heights at $100 \mathrm{mb}$ were extracted from each profile. Data for the radiosonde that did not reach above $100 \mathrm{mb}$ were excluded from considerations. $12 \mathrm{~h}$ data were used to create the daily averages for atmospheric pressure, level of pion production, temperature at that level, and muon measurements. To remove the effect of the diurnal and possible 27-day variations, daily data are then averaged to calculate the monthly means. Moreover, data for the period of solar flares, magnetic storms, and Forbush decreases were excluded. Table 1 presents some of the statistical parameters for the considered variables.

The relationship between the three parameters and the cosmic ray muons was investigated on the basis of the effective level of generation method [21]. This method is a combination of Duperier's [20] and Blackett's [22] methods, in which the muon intensity at the detection level was related to the atmospheric pressure, level of pion production, and the temperature at that level using the following expression:

$$
\frac{I-I_{0}}{I_{0}}=\alpha\left(P-P_{0}\right)+\beta\left(T-T_{0}\right)+\gamma\left(H-H_{0}\right),
$$

where $I_{0}, P_{0}, T_{0}$, and $H_{0}$ are the mean values of intensity, pressure, temperature, and production level for the considered period. $\alpha, \beta$, and $\gamma$, are the pressure, temperature, and height coefficients, respectively.

The first term of (1) represents the effect of the atmospheric mass above the detector, whereas the second indicates the influence of atmospheric temperature at the pion production level. The final term shows the dependence of the surviving muons on the distance between the production level, usually taken as $100 \mathrm{mb}[9,14]$, and the detection level.

Regression analysis between cosmic ray muon intensities and these parameters was performed and the three coefficients $(\alpha, \beta$, and $\gamma)$ were calculated. Corrections to the muon data using the obtained coefficients are then conducted with the aid of (1).

\section{Results and Discussion}

Figure 1 presents yearly fluctuations in (a) atmospheric pressure, (b) level of maximum production, (c) temperature at that level, and the (d) muon counts, from their mean values. It can be seen that the three atmospheric parameters have seasonal variations, which in turn affect the muon rates, as clearly seen in Figure 2.

Figure 3 shows scatterplots that indicate the relationship between the raw muon rate and each individual parameter. Both atmospheric pressure and temperature at the $100 \mathrm{mb}$ level are inversely correlated with the muon rates. On the contrary, the muon rate is directly proportional to the level of pion production. The spread in the data is due to several causes. For instance, the 12-hour separation between the radiosonde flights may give some experimental error on the measured values.

Since atmospheric pressure is the most effective parameter, we first correct the muon rates for this parameter. The regression results between pressure and the muon rate (Figure 3(a)) for the data considered give a barometric coefficient value of $-0.17 \pm 0.05 \% / \mathrm{mb}$. This value did not differ greatly from that obtained in our previous study [23]. Figure 4 shows the relationship between the pressure-corrected muon rate and (a) level of pion production and (b) the temperature at that level.

It can be seen that the dependence of cosmic ray muons on the height and the temperature found in Figure 3 is critically distorted by the dependence on the pressure. The dependence of the pressure-corrected muon rate on these two parameters shows the opposite relationship compared to the uncorrected rate. The muon rate is inversely correlated with the level of muon production. This means that, as the production level becomes higher, the observed muons at the detection level will be lower. On the other hand, the corrected muon rate correlates with temperature at the production level. This implies that, during cold times, pions will be less likely to decay to produce muons before they interact. The opposite holds for warm times.

Having established the effect of each individual variable on the muon rate, our next step was to conduct regression analysis between muon rate and different combinations of the three parameters. The calculation procedures were conducted on the basis of (1). Multiple regression analyses were conducted between (i) raw muon rate and both pressure and temperature at the production level; (ii) raw muon rate and both pressure and level of pion production; (iii) raw muon rate and the three parameters; and (iv) pressure-corrected muon rate, using the method discussed in Maghrabi et al. [23] and Maghrabi et al. [12,13], which was correlated with both height and the temperature at the production level. $12 \mathrm{~h}$ data for each 
TABLE 1: Summary of the mean values of the parameters for the considered period.

\begin{tabular}{lccccc}
\hline & Min. & Max. & Mean & Skewness & Kurtosis \\
\hline Height $[\mathrm{km}]$ & 16.38 & 16.84 & $16.60 \pm 0.01$ & 0.22 & -1.31 \\
Pressure $[\mathrm{mb}]$ & 933.64 & 955.43 & $942.99 \pm 0.49$ & -0.13 & -0.99 \\
$T\left[{ }^{\circ} \mathrm{C}\right]$ & -78.82 & -70.11 & $-74.79 \pm 0.24$ & 0.10 & -1.14 \\
Rate $[$ counts/15 min] & 157.49 & 161.34 & $159.41 \pm 0.10$ & 0.02 & -0.98 \\
\hline
\end{tabular}

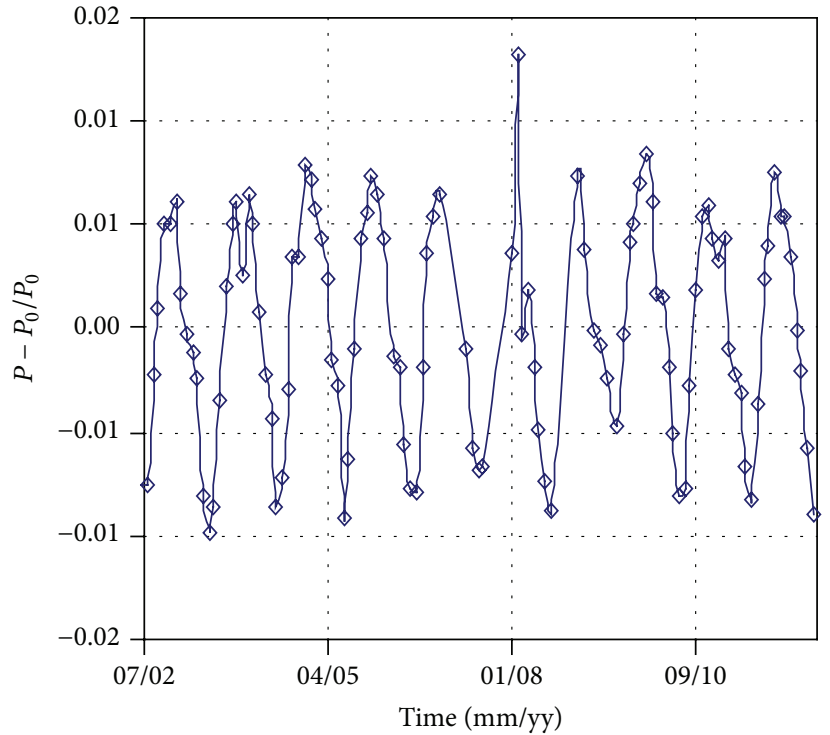

(a)

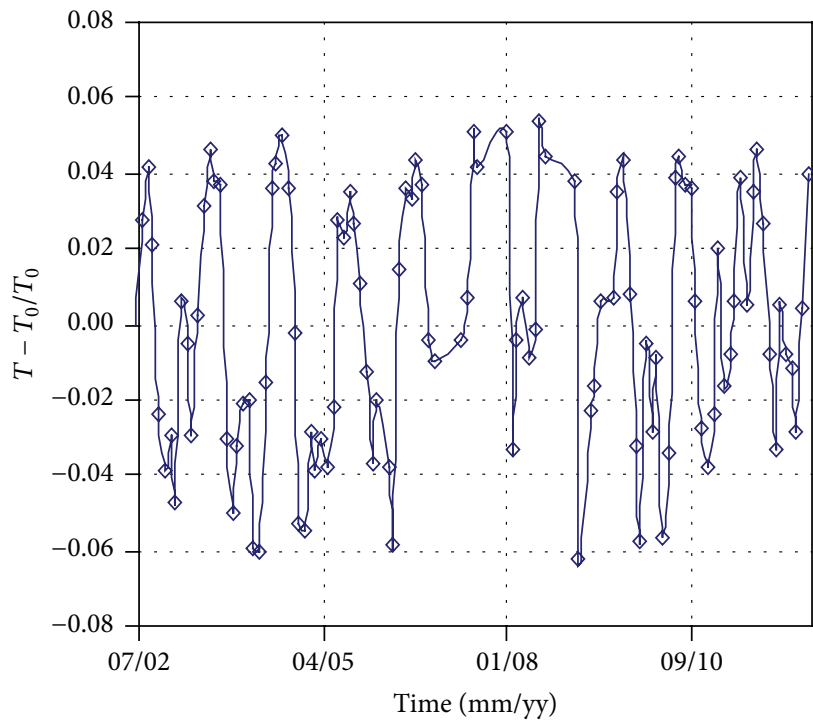

(c)

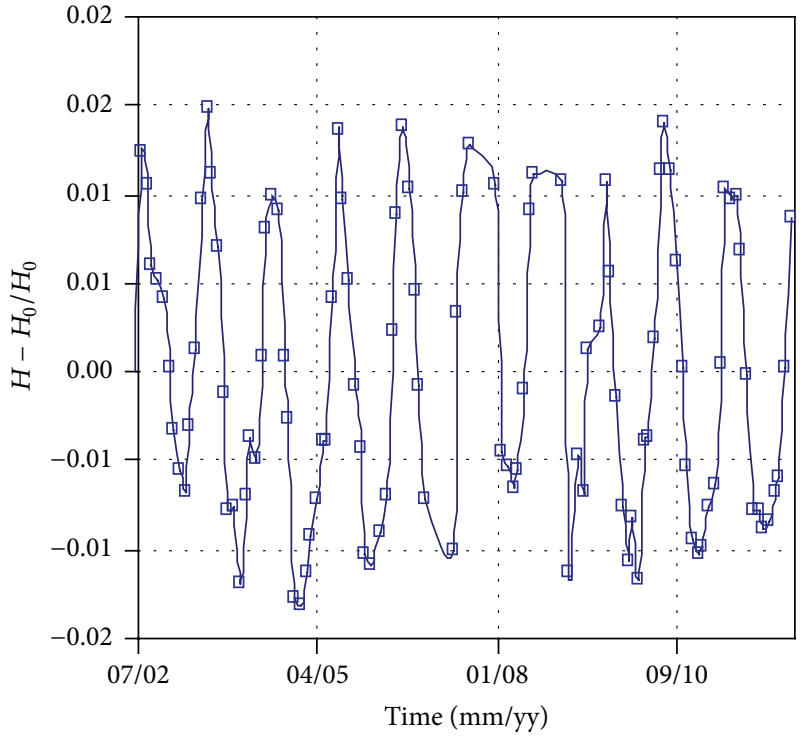

(b)

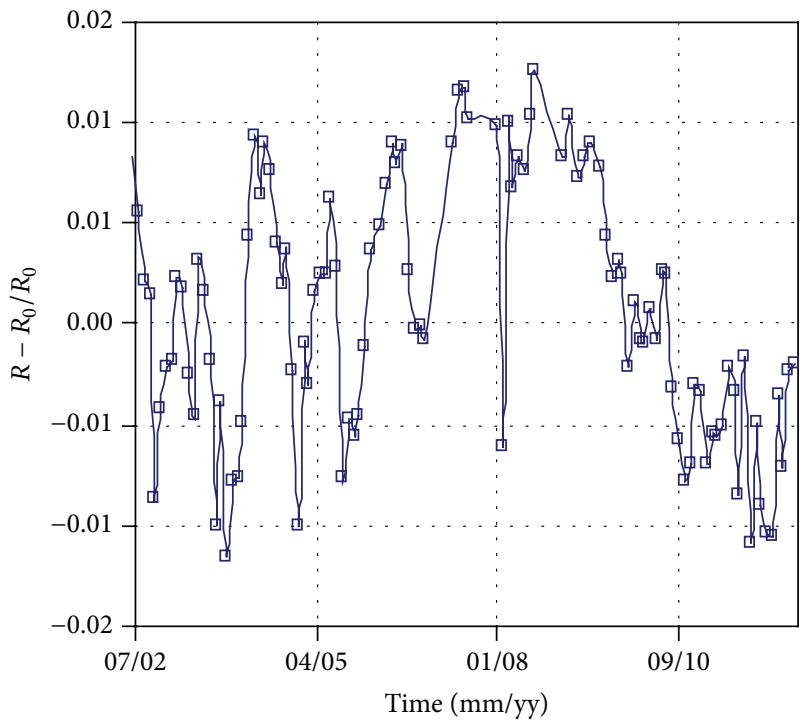

(d)

FIGURE 1: Time series of deviations of the (a) atmospheric pressure, (b) level of maximum production, (c) temperature at that level, and (d) muon counts, from their mean values during the 11 years.

day during the specific month were used in the calculations. For each month, the regression and the correlation coefficients were obtained.

Figure 5 shows the distribution of the calculated coefficients for $(\alpha, \beta),(\alpha, \gamma),(\alpha, \beta, \gamma)$, and $(\beta ; \gamma)$; their maximum, minimum, and mean values are given in Table 2 .
For the first combination $(P, T)$, the pressure coefficient, $\alpha$, was between -0.300 and -0.001 with a mean value of -0.151 $\pm 0.006 \% / \mathrm{mb}$. The temperature coefficient $\beta$ ranged between -0.230 and 0.270 with a mean of $0.030 \pm 0.007 \% /{ }^{\circ} \mathrm{C}$. In the correlations between $(P, H)$ and muon rate, $\alpha$ did not change much from the previous set. The level of the pion production 


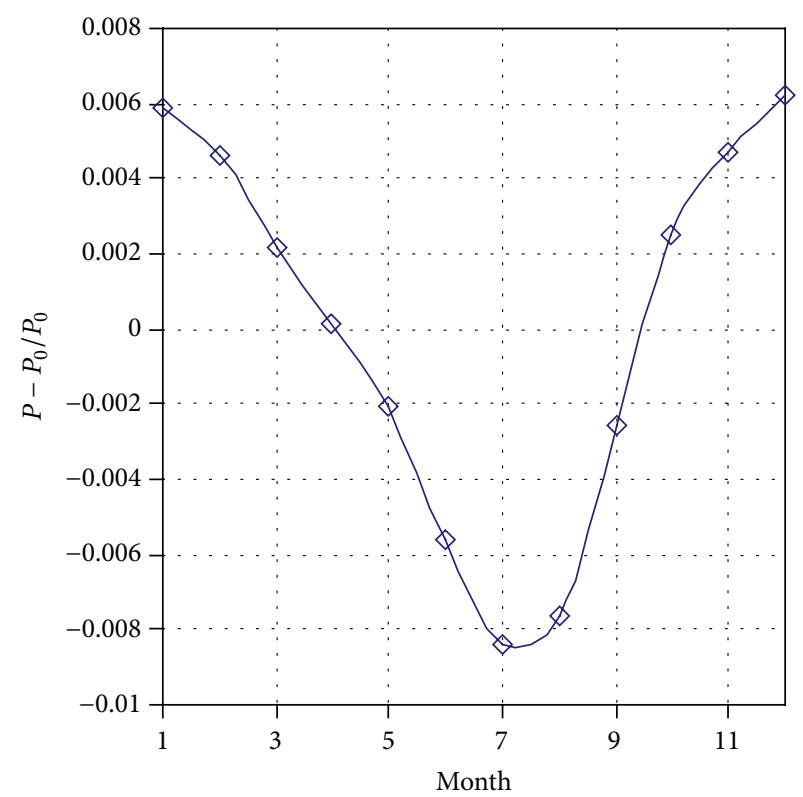

(a)

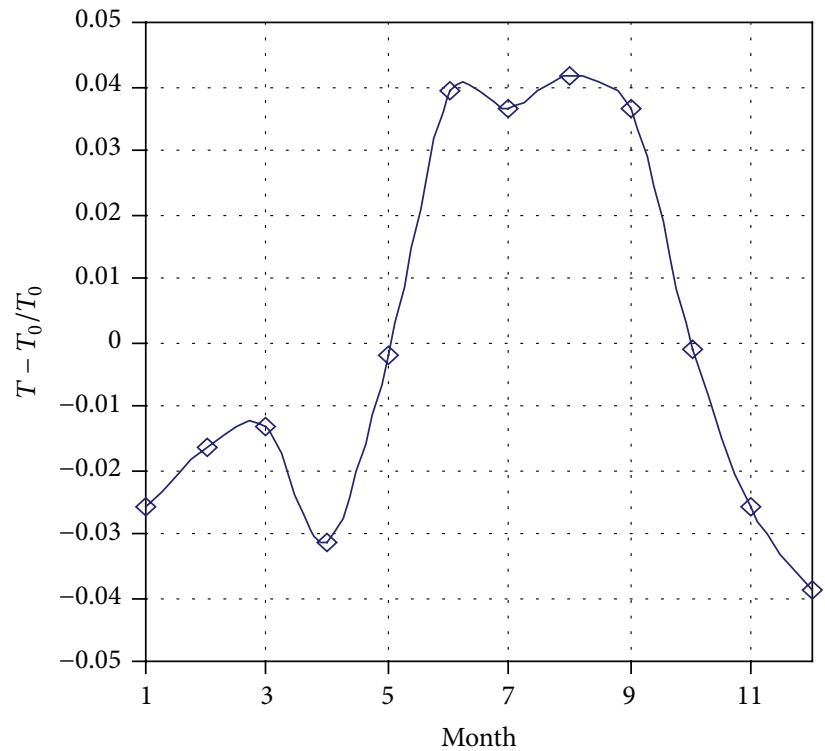

(c)

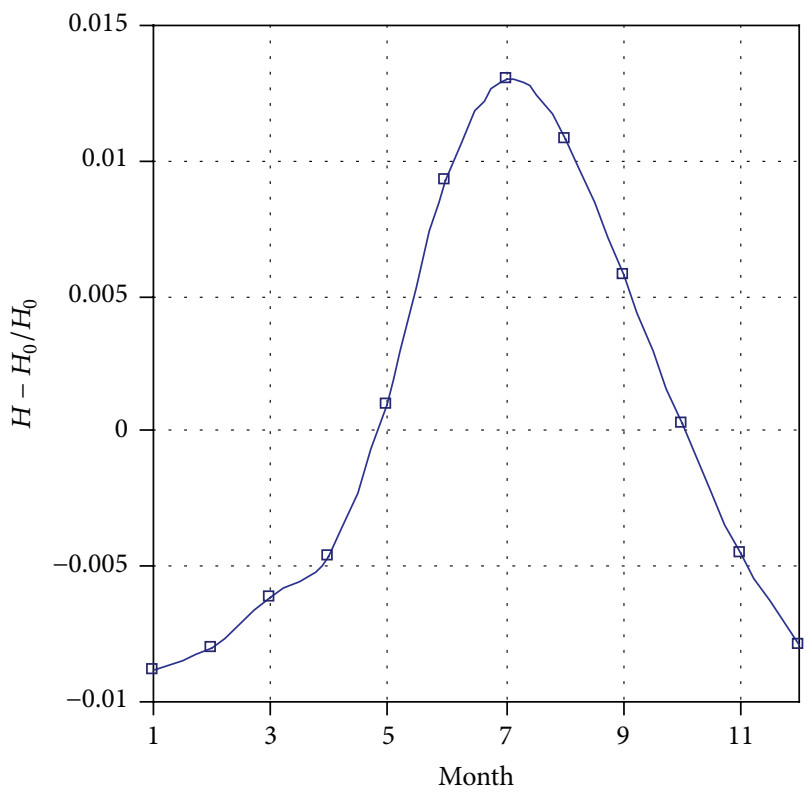

(b)

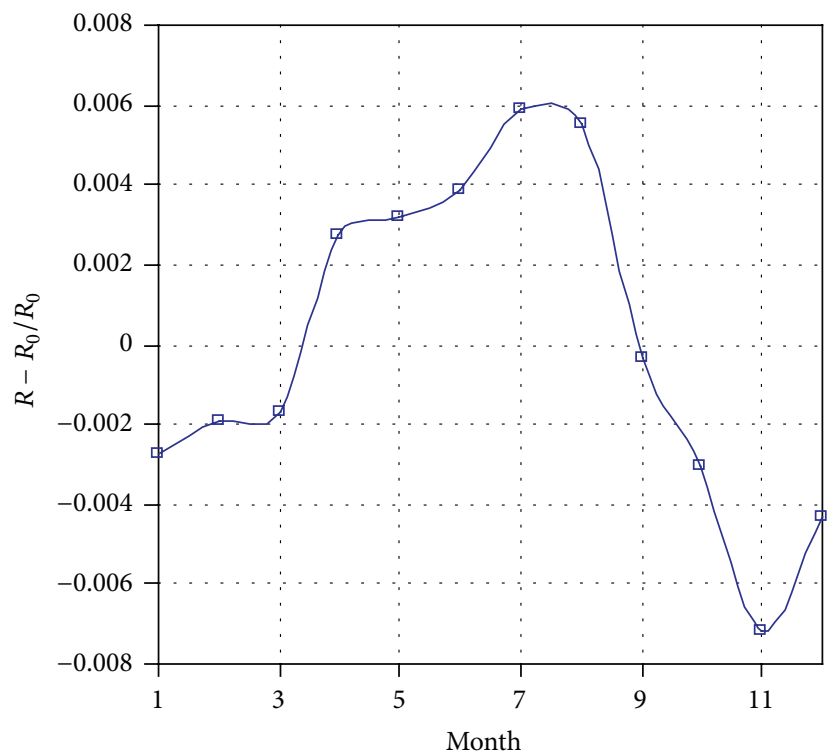

(d)

FIGURE 2: Monthly variations in (a) atmospheric pressure, (b) level of maximum production, (c) temperature at that level, and (d) muon counts, from their 11-year mean values.

coefficient $\gamma$ varies between -12.100 and 0.460 with a mean of $-4.876 \pm 0.352 \% / \mathrm{km}$. For correlation between the muon rate and the three parameters, $\alpha, \beta$, and $\gamma$ were $-0.151 \pm$ $0.005 \% / \mathrm{mb}, 0.019 \pm 0.007 \% /{ }^{\circ} \mathrm{C}$, and $-1.644 \pm 0.238 \% / \mathrm{km}$, respectively. In the case of multiple regression between the pressure-corrected muon rate and both $(T, H)$ (Figure $5(\mathrm{~d})$ ), the temperature coefficient, $\beta$, was between -0.019 and 0.260 with a mean of $0.020 \pm 0.006 \% /{ }^{\circ} \mathrm{C}$. The level of the pion production coefficient $\gamma$ lies between -10.70 and 5.91 with a mean of $-1.28 \pm 0.321 \% / \mathrm{km}$.

Figure 6 shows monthly averages of muon measurements for the whole period corrected for (a) pressure and temperature, (b) pressure and height, (c) for the three parameters, and (d) pressure-corrected muons correlated with both temperature and height. The uncorrected muon rate was plotted for comparison purposes.

The uncorrected rate increases from January to March and May to July. It shows a decreasing trend during August to December. The two-parameter-corrected rate is clearly in an inverse relationship with the uncorrected rate for the entire considered period. In the case of the three-parameter corrections, the corrected rate continues to increase from May to January and decreases for the other months. The same behavior of the three parameters corrections is seen in 


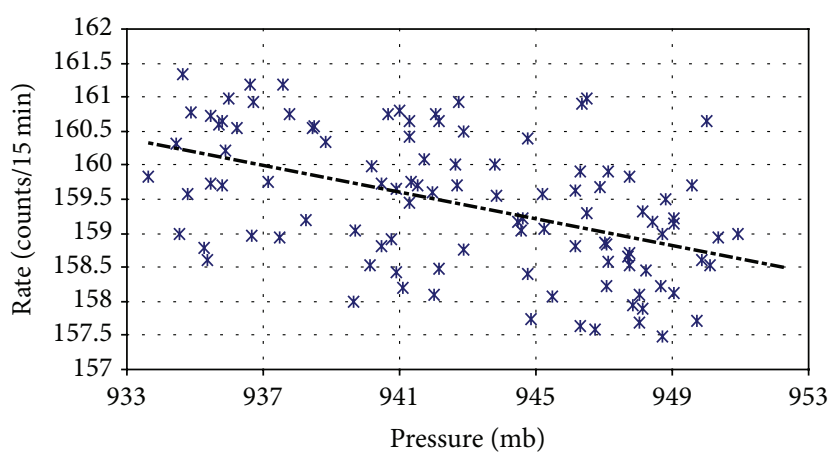

(a)

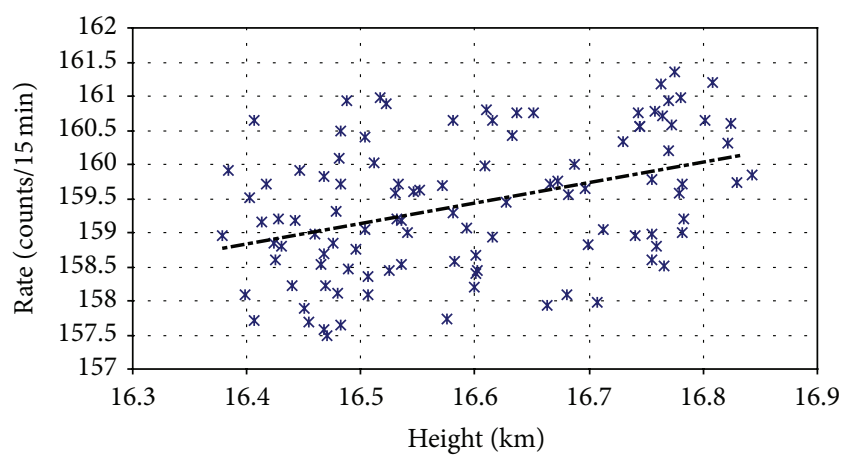

(b)

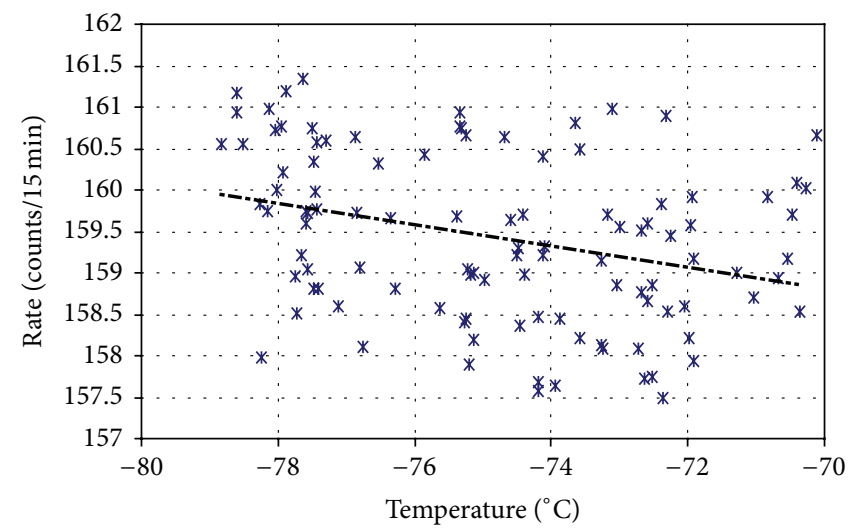

(c)

FiguRE 3: Scatterplot between the muon mean monthly values against (a) pressure, (b) height, and (c) temperature. The dashed thick lines are the regression lines.

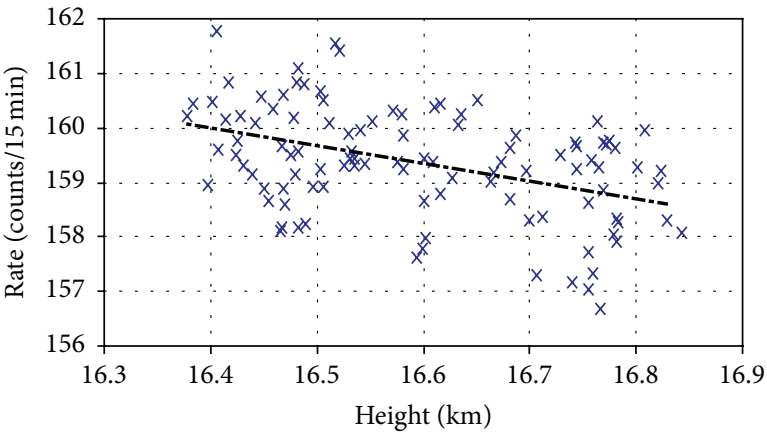

(a)

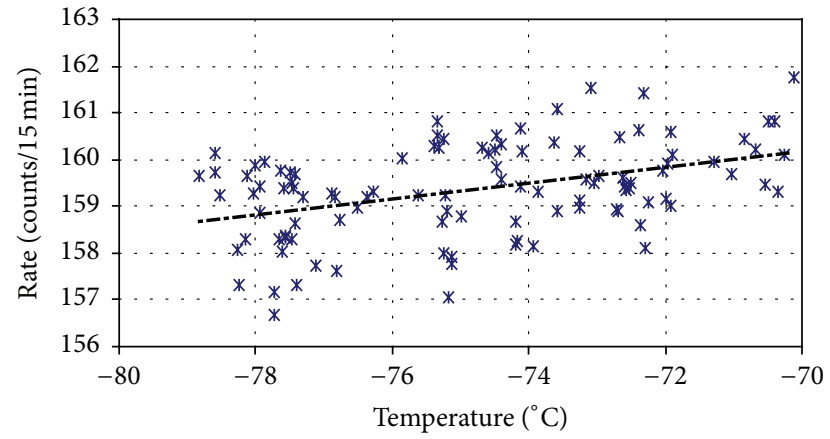

(b)

FIGURE 4: Scatterplot between the pressure-corrected muon rate and (a) level of pion production and (b) temperature at that level. The dashed thick lines are the regression lines.

the corrections using, last case, pressure-corrected muons correlated with both temperature and height. It is clearly obvious that although the corrections have been made to the muon rate using two or three parameters, seasonal variations in the muon rate are evident. This means that there is another cause, either terrestrial and/or extraterrestrial, for this seasonality rather than the three considered parameters. Moreover, the fact that the long-term variation does not depend on season, but in a random way similar to a seasonal variation, is another possible explanation for this seasonality.
To investigate this seasonality further, the whole dataset (2002-2012) was divided into four groups: December, January, and February representing winter, March, April, and May spring, June, July, and August summer, and September, October, and November fall.

Similar procedures were applied for each season. Regression analysis between the raw muon rates and $(P$ and $H)$, ( $P$ and $T$ ), the three parameters, and between pressurecorrected muon rate and $(H, T)$ was carried out; the obtained correction coefficients are given in Table 3 . It can be seen that 

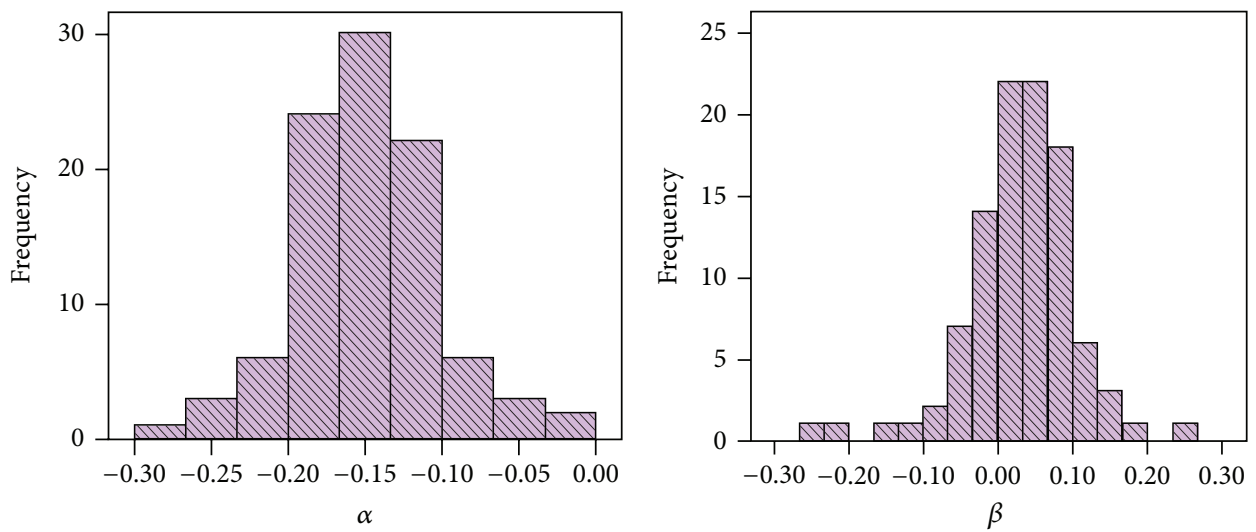

(a)
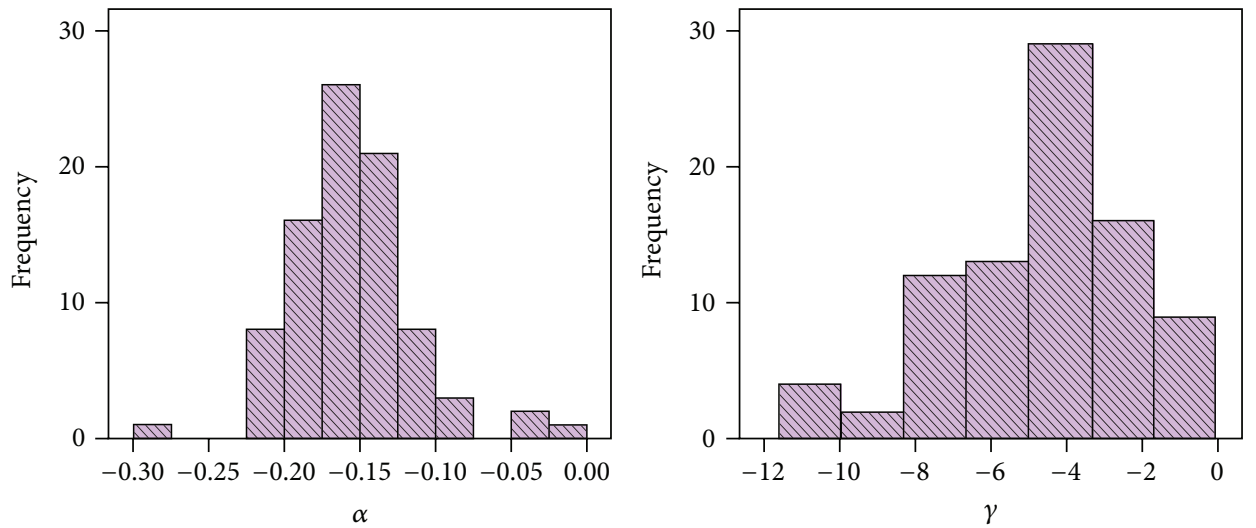

(b)
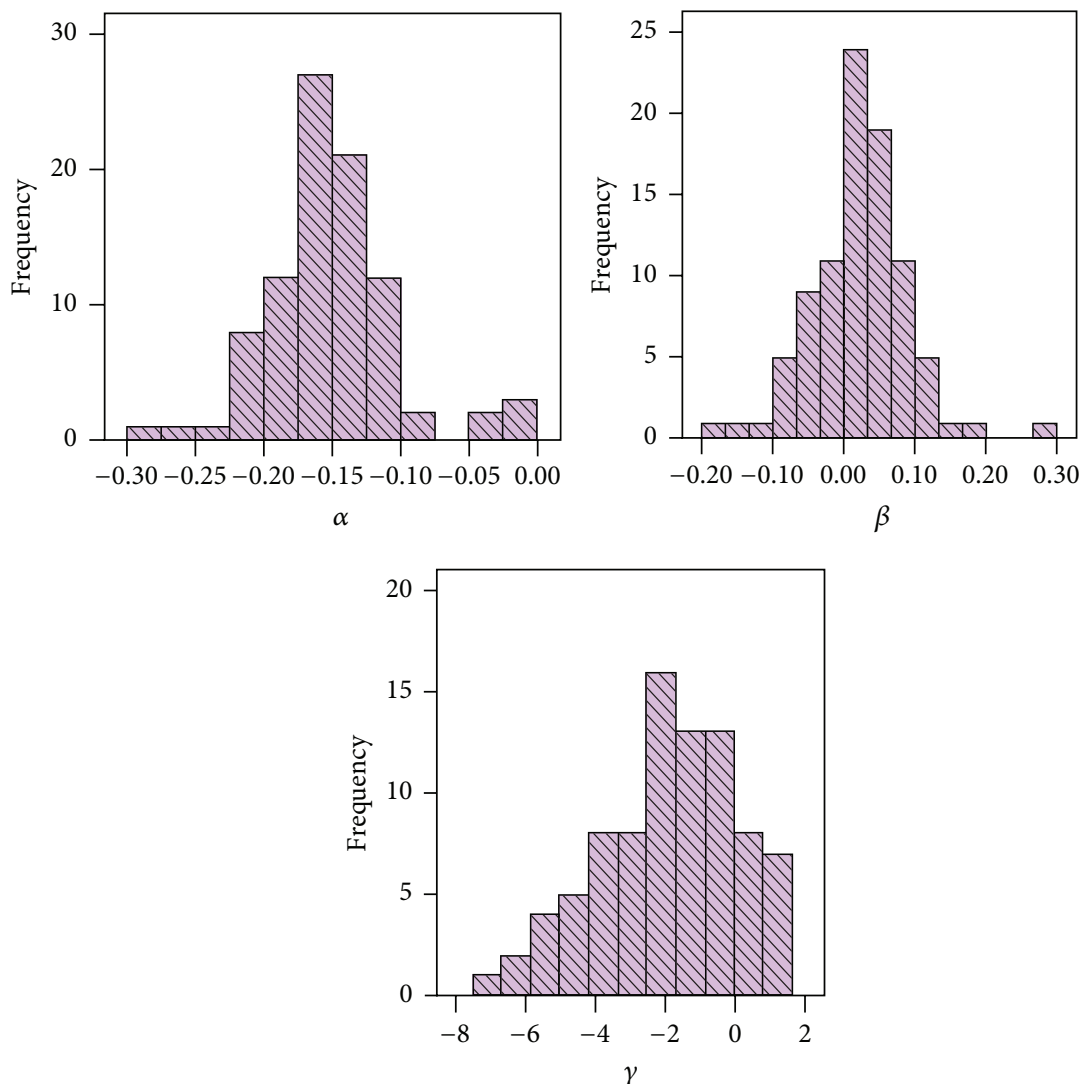

(c)

FIgUre 5: Continued. 

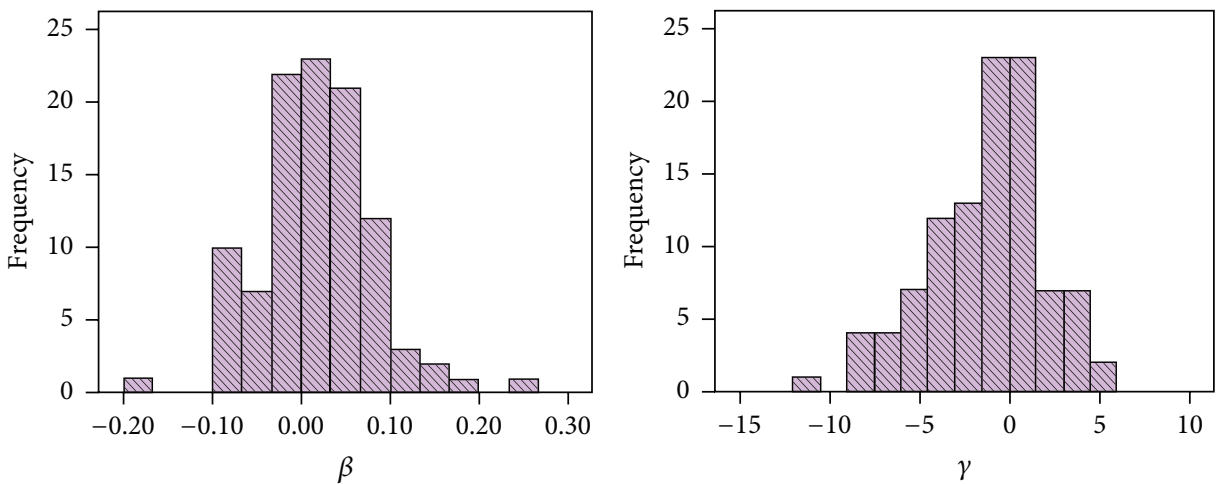

(d)

Figure 5: Frequency distribution of the calculated coefficients for (a) $\alpha$ and $\beta$, (b) $\alpha$ and $\gamma$, (c) $\alpha$, $\beta$, and $\gamma$, and (d) $\gamma$ and $\beta$ obtained from the regression analysis between pressure-corrected muon rate and both the level and the temperature at the production level.

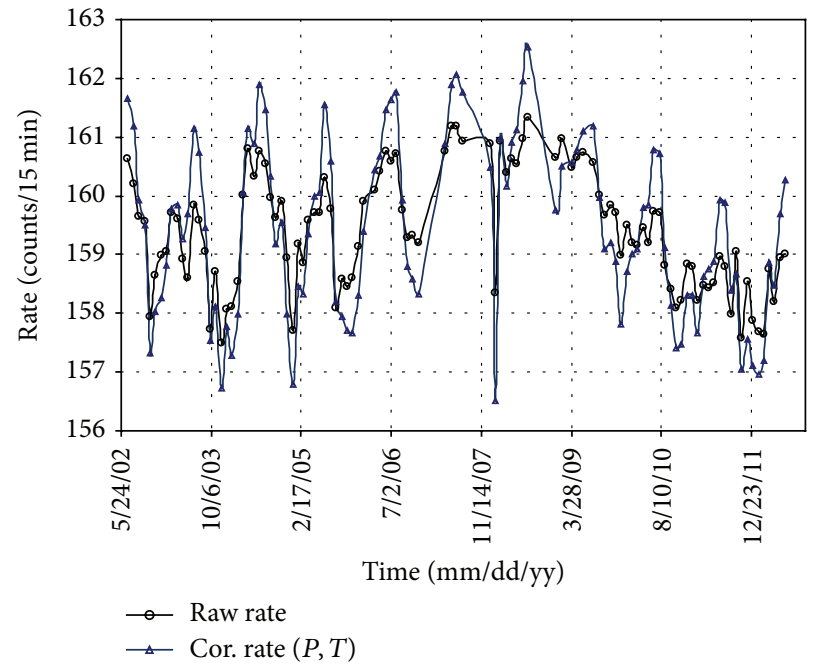

(a)

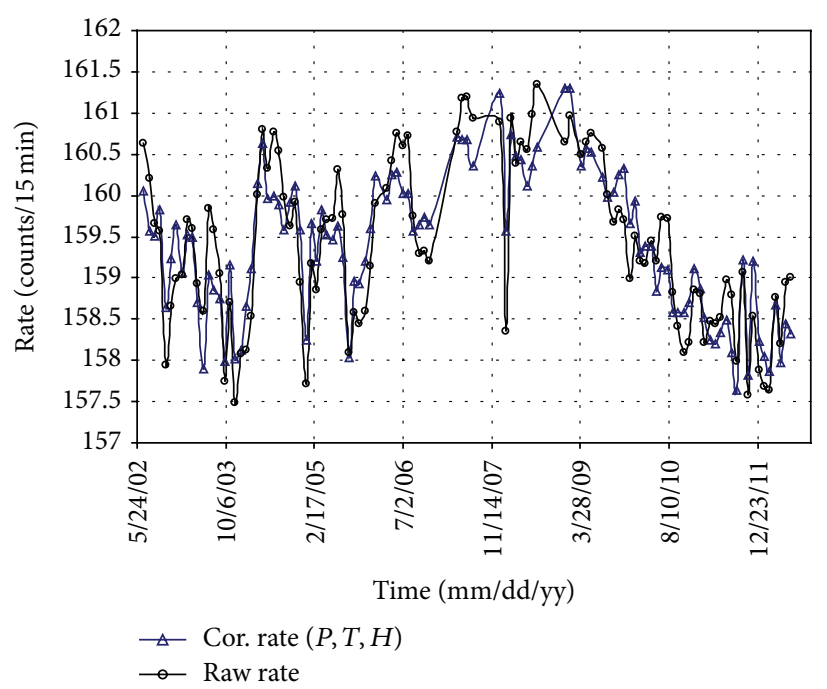

(c)

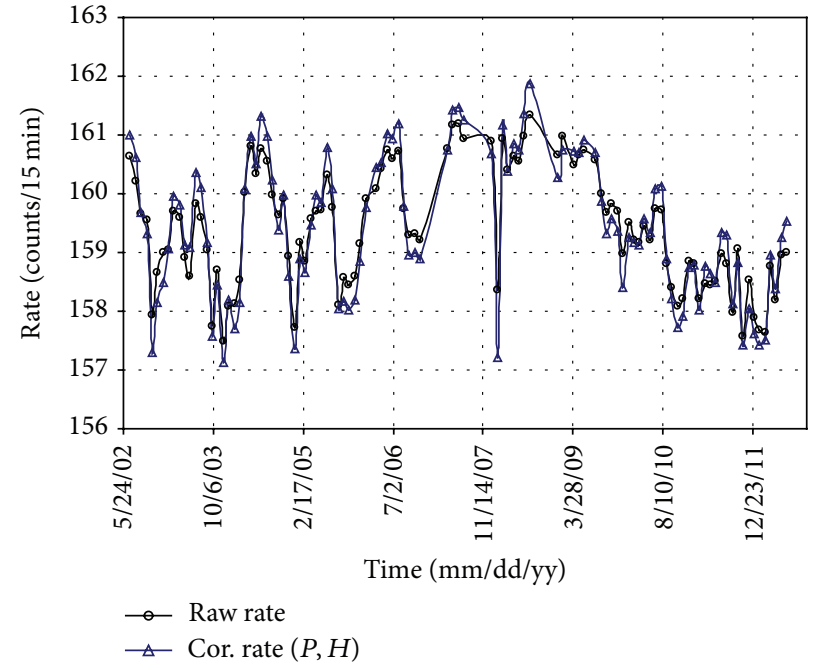

(b)



(d)

FIGURE 6: Example comparing the monthly values of the uncorrected muon rate for the considered period against those corrected for (a) pressure and temperature, (b) pressure and height, (c) the three parameters, and (d) pressure-corrected muons correlated with both temperature and height. 
TABLE 2: Summary of minimum, maximum, and mean values of the obtained coefficients $(\alpha, \beta),(\alpha, \gamma),(\alpha, \beta, \gamma)$, and $(\beta, \gamma)$.

\begin{tabular}{lccc}
\hline & Min. & Max. & Mean \\
\hline$\alpha$ & -0.300 & -0.001 & $-0.151 \pm 0.006$ \\
$\beta$ & -0.230 & 0.270 & $0.030 \pm 0.007$ \\
\hline$\alpha$ & -0.310 & 0.010 & $-0.152 \pm 0.005$ \\
$\gamma$ & -12.100 & 0.460 & $-4.876 \pm 0.352$ \\
\hline$\alpha$ & -0.290 & -0.010 & $-0.151 \pm 0.005$ \\
$\beta$ & -0.190 & 0.270 & $0.019 \pm 0.007$ \\
$\gamma$ & -7.140 & 4.840 & $-1.644 \pm 0.238$ \\
$\beta$ & -.019 & 0.26 & $0.02 \pm 0.006$ \\
$\gamma$ & -10.70 & 5.91 & $-1.28 \pm 0.321$ \\
\hline
\end{tabular}

TABLE 3: Mean values of the obtained coefficients $(\alpha, \beta),(\alpha, \gamma),(\alpha$, $\beta, \gamma)$, and $(\beta, \gamma)$ for the four seasons.

\begin{tabular}{ccccc}
\hline & Summer & Winter & Fall & Spring \\
\hline$\alpha$ & -0.09 & -0.11 & -0.08 & -0.13 \\
$\beta$ & -0.06 & 0.08 & -0.01 & 0.04 \\
\hline$\gamma$ & -0.74 & -1.29 & -0.51 & -1.31 \\
$\alpha$ & -0.07 & -0.11 & -0.09 & -0.14 \\
\hline$\gamma$ & -1.27 & -0.81 & -0.61 & -0.87 \\
$\alpha$ & -0.07 & -0.11 & -0.09 & -0.14 \\
$\beta$ & -0.09 & 0.07 & -0.01 & 0.03 \\
\hline$\beta$ & -0.09 & 0.07 & -0.01 & 0.03 \\
$\gamma$ & -1.57 & -0.81 & -0.69 & -0.41 \\
\hline
\end{tabular}

the values of $(\alpha, \beta)$ did not change over the seasons. However, the sign of the temperature coefficient is negative in both summer and fall and positive in winter and spring. For the $(P, H)$ combination, the $\alpha$ values were almost the same for all seasons. In winter and spring, the $\gamma$ values are higher compared to those for summer and fall. In the case of the three parameters, the pressure and the temperature coefficients change very slightly between the seasons. The $\gamma$ coefficient has almost the same values in both winter and spring, $-1.27 \% \mathrm{~km}^{-1}$ in summer and $-0.6 \% \mathrm{~km}^{-1}$ in winter. The temperature coefficient $\beta$, as in the case of the two parameters, positively affects the rate in winter and spring and negatively during the other seasons.

Variations of the sign of the temperature coefficients $\beta$ between the seasons have not been previously reported for this particular cut-off rigidity and for such a long term of the measurements. We suggest that long-term measurements from other muon detectors operating at different cut-off rigidities may be used for future investigations. Also, quasiperiodicity investigations between the cosmic ray muon data presented here and several atmospheric variables are suggested, for searching for such variations.

\section{Conclusions}

In this study, cosmic ray data from the KACST muon detector and radiosonde measurements (Riyadh, Saudi Arabia; Rc = 14.4 GV) for the period 2002-2012 were used to study the effect of atmospheric pressure, level of pion production, and temperature at that level on the cosmic ray muons.

The results show that while the raw muon rate is directly correlated with the level of pion production it is inversely correlated with the other two variables. Muon data are then pressure corrected and correlated with the other two variables. The dependence of the pressure-corrected muon rate on both variables was reversed compared to the uncorrected rate.

Regression analysis between muon rate and four combinations of the three parameters was then carried out on the basis of the Duperier and Blackett methods. These are (i) raw muon rate correlated with both pressure and temperature at production level; (ii) raw muon rate correlated with pressure and level of pion production; (iii) raw muon rate correlated with the three parameters; and (iv) pressure-corrected muon rate correlated with both level and the temperature at that production level. For each correlation, the corresponding coefficients are obtained and their distributions were studied. The obtained coefficients were used to correct the measured muon rates. We found that, even if corrections were made to the detected muons using these three variables, seasonal variations are evident. This was in disagreement with results previously established by several investigators. We suggest that other terrestrial and/or extraterrestrial causes must be considered. Long-term variation that does not depend on season, but in a random way similar to seasonal variation, is another possible explanation for this seasonality. To investigate this seasonality further, the dataset was divided into four seasonal groups, and the same four correlations were carried out. The analysis showed that the temperature at the pion production level positively affects the muons in spring and winter and negatively (i.e., inversely correlated) in fall and summer. No definite explanations for the variations of the sign of the temperature coefficients between the seasons can be reached yet.

\section{Competing Interests}

The authors declare that they have no competing interests.

\section{Acknowledgments}

The authors would like to thank King Abdulaziz City for Science and Technology (KACST) for supporting this work.

\section{References}

[1] H. Svensmark and E. Friis-Christensen, "Variation of cosmic ray flux and global cloud coverage-a missing link in solar-climate relationships," Journal of Atmospheric and SolarTerrestrial Physics, vol. 59, no. 11, pp. 1225-1232, 1997.

[2] K. Kudela, M. Storini, M. Y. Hofer, and A. Belov, "Cosmic rays in relation to space weather," Space Science Reviews, vol. 93, no. 1-2, pp. 153-174, 2000.

[3] D. R. Kniveton and M. C. Todd, "On the relationship of cosmic ray flux and precipitation," Geophysical Research Letters, vol. 28, no. 8, pp. 1527-1530, 2001.

[4] M. Pudovkin, "Influence of solar activity on the lower atmosphere state," International Journal of Geomagnetism and Aeronomy, vol. 5, no. 2, pp. 1-18, 2004. 
[5] L. I. Dorman, "Long-term cosmic ray intensity variation and part of global climate change, controlled by solar activity through cosmic rays," Advances in Space Research, vol. 37, no. 8, pp. 1621-1628, 2006.

[6] K. Kudela and M. Storini, "Possible tools for space weather issues from cosmic ray continuous records," Advances in Space Research, vol. 37, no. 8, pp. 1443-1449, 2006.

[7] K. Maeda and M. Wada, "Atmospheric temperature effect upon the cosmic ray intensity at sea level," Journal of the Scientific Research Institute, Tokyo, vol. 48, pp. 71-79, 1954.

[8] L. Dorman, Cosmic Rays in the Earth's Atmosphere and Underground, Kluwer Academic, New York, NY, USA, 2004.

[9] C. R. Braga, A. Dal Lago, T. Kuwabara, N. J. Schuch, and K. Munakata, "Temperature effect correction for the cosmic ray muon data observed at the Brazilian Southern Space Observatory in São Martinho da Serra," Journal of Physics: Conference Series, vol. 409, no. 1, Article ID 012138, 2013.

[10] A. Berkova, R. Clay, E. Eroshenko, and V. Yanke, "Atmospheric variations as observed by Adelaide and Buckland Park muon telescopes," in Proceedings of the 33rd International Cosmic Ray Conference (ICRC '13), pp. 2-9, Rio De Janerio, Brazil, July 2013.

[11] C. Rogelio and J. Valdés-Galicia, "Variations in cosmic radiation intensity associated with the barometric effect," GeofisicaInternacional, vol. 39, no. 1, pp. 135-137, 2000.

[12] A. Maghrabi, M. Almutayri, H. Al Harbi, and M. Baig, "Atmospheric-weighted temperature and its influence on cosmic ray muons," in Proceedings of the 34rd International Cosmic Ray Conference (ICRC '15), The Hague, The Netherlands, August 2015.

[13] A. H. Maghrabi, R. N. Alotaibi, M. M. Almutayri, and M. S. Garawi, "Influence of the atmospheric mass on the high energy cosmic ray muons during a solar cycle," Advances in Astronomy, vol. 2015, Article ID 939146, 7 pages, 2015.

[14] M. Ganeva, S. Peglow, R. Hippler, M. Berkova, and V. Yanke, "Seasonal variations of the muon flux seen by muon telescope MuSTAnG," Journal of Physics: Conference Series, vol. 409, no. 1, Article ID 012242, 2013.

[15] R. R. S. De Mendonça, J.-P. Raulin, E. Echer, V. S. Makhmutov, and G. Fernandez, "Analysis of atmospheric pressure and temperature effects on cosmic ray measurements," Journal of Geophysical Research: Space Physics, vol. 118, no. 4, pp. 14031409, 2013.

[16] A. N. Dmitrieva, I. I. Astapov, A. A. Kovylyaeva, and D. V. Pankova, "Temperature effect correction for muon flux at the Earth surface: estimation of the accuracy of different methods," Journal of Physics: Conference Series, vol. 409, no. 1, Article ID 012130, 2013.

[17] A. Maghrab, A. Alghamdi, R. Alotaibi, M. Almutariand, and M. Garawi, "On the calibration of a single channel cosmic ray particle detector," in Proceedings of the High Energy, Optical, and Infrared Detectors for Astronomy VI, vol. 9154 of Proceedings of SPIE, Montréal, Canada, July 2014.

[18] A. Maghrabi, R. Al Oataibi, M. Almotery, and M. Garawi, "The temperature effect on cosmic-ray intensity as observed at Mid latitude City," in Proceedings of the 24th European Cosmic Ray Symposium, Christian-Albrechts-Universitaetzu, Kiel, Germany, September 2014, S2-549 CRs at Earth and Planets (GEO) Invited Talk.

[19] V. M. Dvornikov, Y. Y. Krestyannikov, and A. Sergeev, "Determination of the mass-average temperature on the cosmic ray intensity data," Geomagnetism and Aeronomy, vol. 16, no. 5, pp. 923-925, 1976.
[20] A. Duperier, "The temperature effect on cosmic-ray intensity and the height of meson formation," Proceedings of the Physical Society, vol. 61, no. 1, pp. 34-40, 1948.

[21] M. D. Berkova, A. V. Belov, E. A. Eroshenko, and V. G. Yanke, "Temperature effect of the muon component and practical questions for considering it in real time," Bulletin of the Russian Academy of Sciences: Physics, vol. 75, no. 6, pp. 820-824, 2011.

[22] P. M. S. Blackett, "On the instability of the barytron and the temperature effect of cosmic rays," Physical Review Letters, vol. 54, no. 11, pp. 973-974, 1938.

[23] A. H. Maghrabi, H. Al Harbi, Z. A. Al-Mostafa, M. N. Kordi, and S. M. Al-Shehri, "The KACST muon detector and its application to cosmic-ray variations studies," Advances in Space Research, vol. 50, no. 6, pp. 700-711, 2012. 

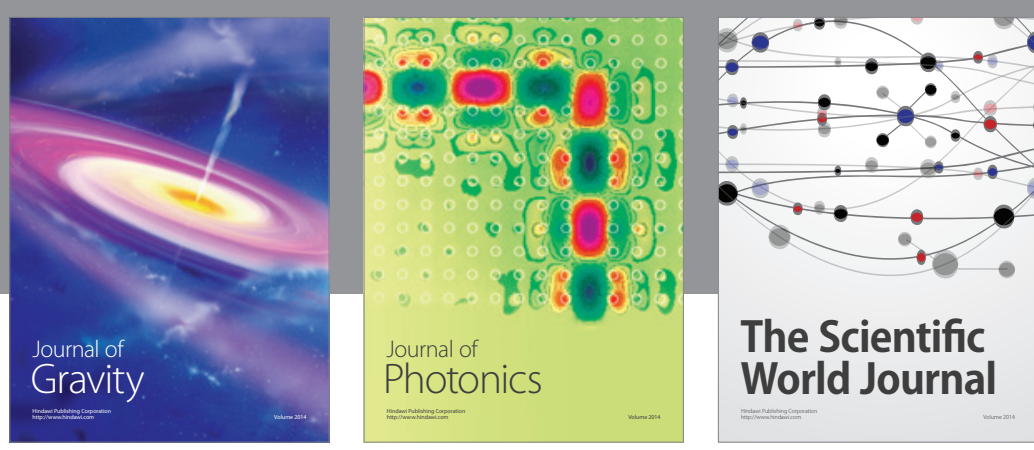

The Scientific World Journal
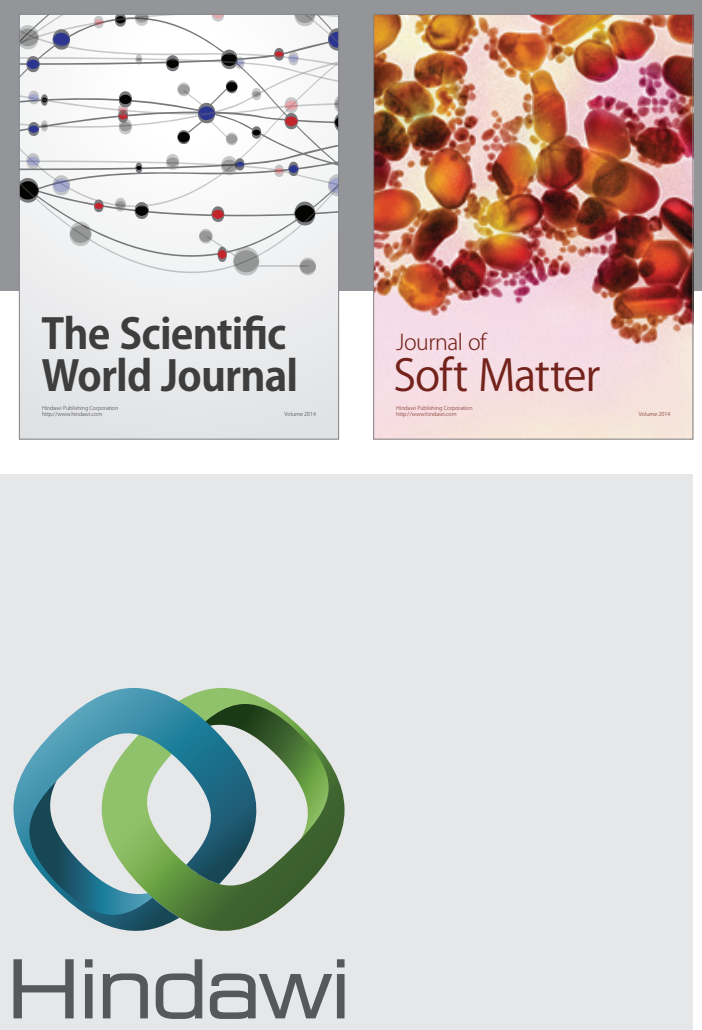

Submit your manuscripts at

http://www.hindawi.com

nternational Journal of

Statistical Mechanics
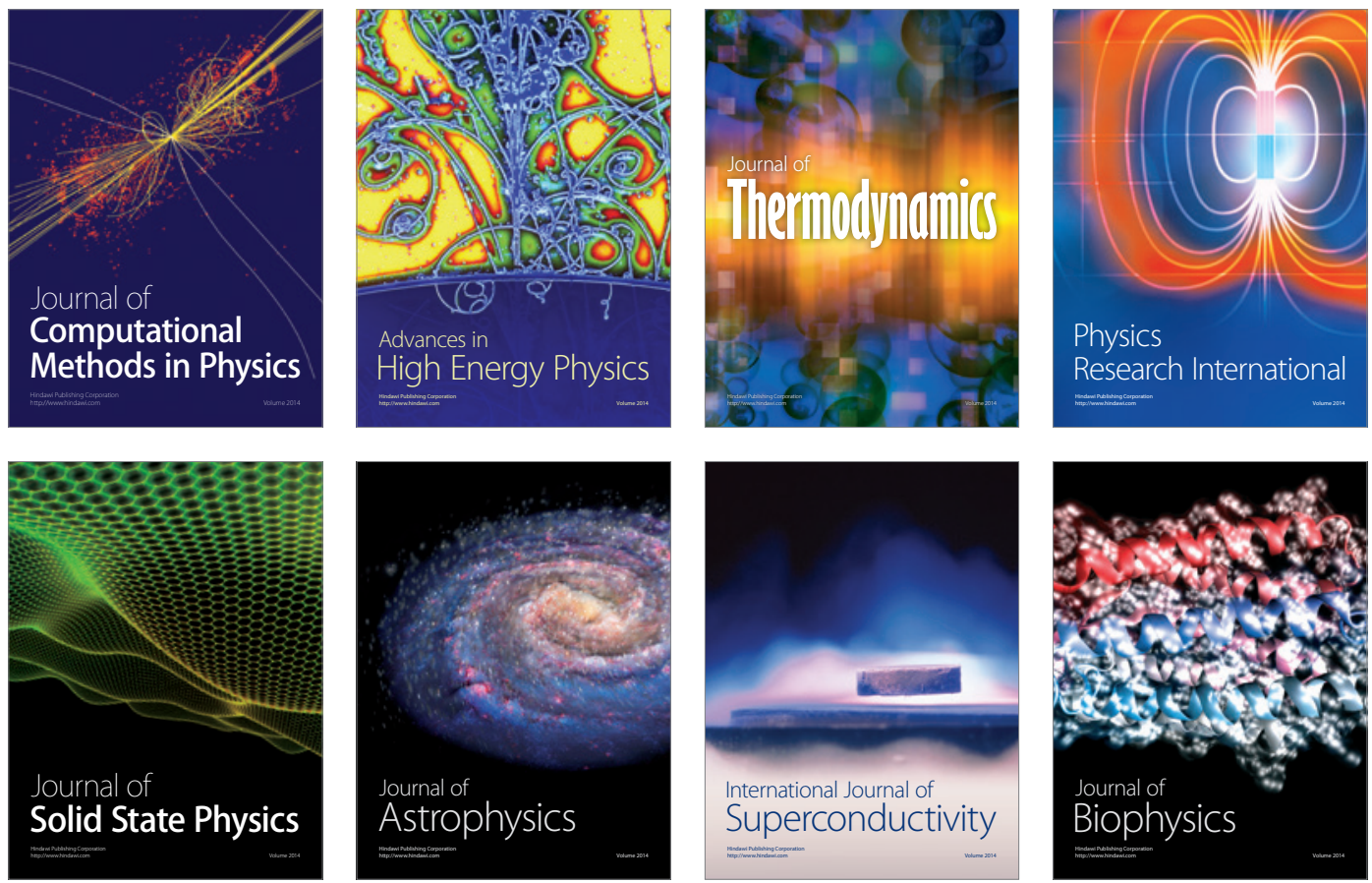
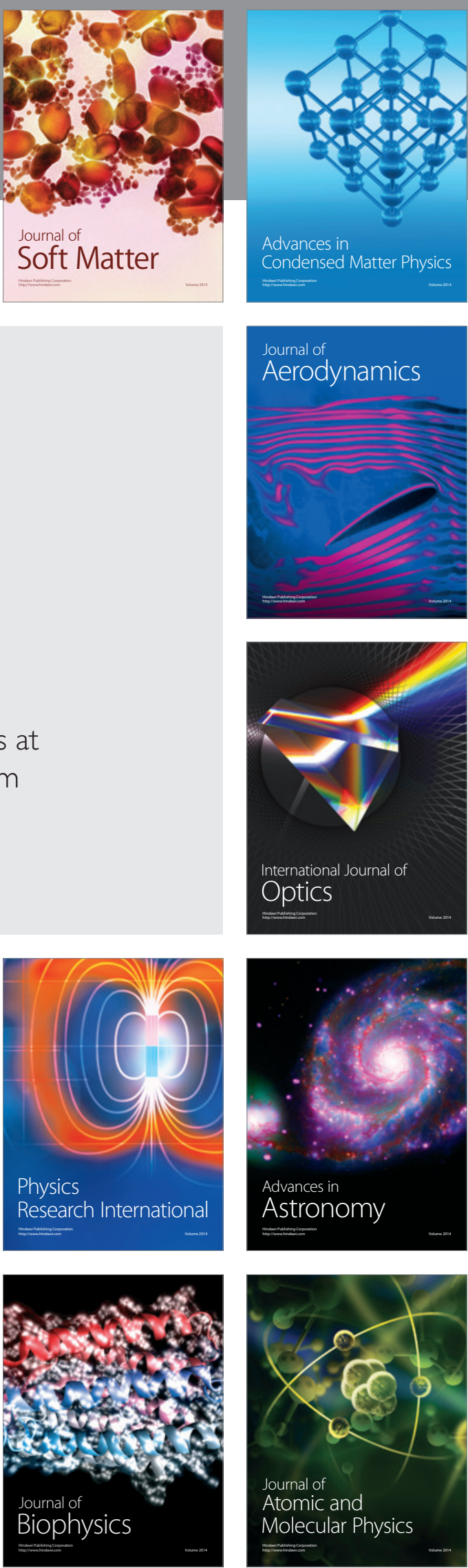